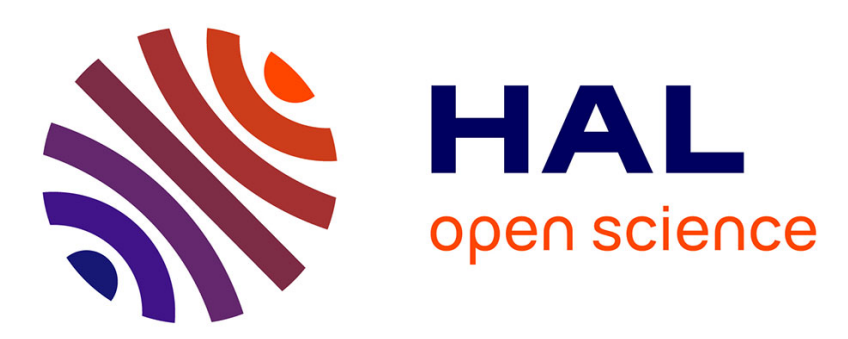

\title{
Ecologie \& Technologies de l'Information et de la Communication (TIC) : une étude exploratoire sur les éco-TIC
}

Sanaa Ait Daoud, Jean Laqueche, Isabelle Bourdon, Florence Rodhain

\section{- To cite this version:}

Sanaa Ait Daoud, Jean Laqueche, Isabelle Bourdon, Florence Rodhain. Ecologie \& Technologies de l'Information et de la Communication (TIC) : une étude exploratoire sur les éco-TIC. Revue management \& avenir, 2010, 10.3917/mav.039.0307 . hal-01965601

\section{HAL Id: hal-01965601 \\ https://hal.science/hal-01965601}

Submitted on 24 Nov 2019

HAL is a multi-disciplinary open access archive for the deposit and dissemination of scientific research documents, whether they are published or not. The documents may come from teaching and research institutions in France or abroad, or from public or private research centers.
L'archive ouverte pluridisciplinaire HAL, est destinée au dépôt et à la diffusion de documents scientifiques de niveau recherche, publiés ou non, émanant des établissements d'enseignement et de recherche français ou étrangers, des laboratoires publics ou privés. 


\section{ECOLOGIE \&AMP; TECHNOLOGIES DE L'INFORMATION ET DE LA COMMUNICATION (TIC) : UNE éTUDE EXPLORATOIRE SUR LES éCO-TIC}

Sanaa Ait-Daoud, Jean Laqueche, Isabelle Bourdon et Florence Rodhain

Management Prospective Ed. | « Management \& Avenir »

2010/9 n³9 | pages 307 à 325

ISSN 1768-5958

Article disponible en ligne à l'adresse :

https://www.cairn.info/revue-management-et-avenir-2010-9-page-307.htm

Distribution électronique Cairn.info pour Management Prospective Ed..

(C) Management Prospective Ed.. Tous droits réservés pour tous pays.

La reproduction ou représentation de cet article, notamment par photocopie, n'est autorisée que dans les limites des conditions générales d'utilisation du site ou, le cas échéant, des conditions générales de la licence souscrite par votre établissement. Toute autre reproduction ou représentation, en tout ou partie, sous quelque forme et de quelque manière que ce soit, est interdite sauf accord préalable et écrit de l'éditeur, en dehors des cas prévus par la législation en vigueur en France. Il est précisé que son stockage dans une base de données est également interdit. 


\title{
Ecologie \& Technologies de I'Information et de la Communication (TIC) : une étude exploratoire sur les éco-TIC
}

\author{
par Sanaa Ait-Daoud ${ }^{97}$, Jean Laqueche ${ }^{98}$, \\ Isabelle Bourdon ${ }^{99}$ et Florence Rodhain ${ }^{100}$
}

Résumé

La question des conséquences négatives des TIC sur l'environnement a suscité l'intérêt de nombreux chercheurs en écologie, en philosophie et en informatique. Cependant, il existe peu de travaux en systèmes d'information traitant cette problématique, d'où l'intérêt de cette recherche. Ce papier expose dans un premier temps une revue de la littérature sur l'impact écologique des TIC et ensuite une étude empirique sur les pratiques mises en place par les entreprises pour réduire l'impact écologique des TIC.

\section{Abstract}

The issue of negative consequences of ICT on the environment has drawn the interest of many researchers in ecology, philosophy and computer science. However, few works in information systems deal with this problem, hence the interest of this research. This paper presents firstly a literature review on the ecological impact of ICT and then an empirical study on practices adopted by companies to reduce the environmental impact of ICT.

Des télégraphes électriques jusqu'aux technologies mobiles, les technologies de l'information et de la communication (TIC) ont joué un rôle important pour faciliter et améliorer la vie des particuliers et des professionnels. Ces technologies ont été considérées essentiellement par rapport aux nombreux avantages qu'elles offrent à la société et à l'économie (Breuil et al., 2008). Grace à ces technologies les coûts de transaction et les frais de transport notamment sont presque nul (Flipo, 2007).

Internet est un des exemples les plus significatifs. Du point de vue économique, Internet a permis à certaines entreprises, spécifiquement aux entreprises de service, de réduire leurs coûts de production et de diminuer, par conséquent, leur coût de revient. Du point de vue social, le réseau numérique a permis aux

97. SANAA AIt -DAOud, Doctorante, Université Montpellier 2, CREGOR, sanae.daoud@yahoo.fr

98. Jean LAqueche, Elève-ingénieur, Ecole Polytechnique Universitaire, CREGO, laqxan@hotmail.com

99. IsABELLE Bourdon, Maitre de conférences-HDR, CREGOR, Université Montpellier 2, isabelle.bourdon@univ-montp2.fr

100. Florence Rodhain, Maître de Conférences-HDR, Université Montpellier 2, rodhain@polytech.univ-montp2.fr 
citoyens du monde de s'ouvrir sur d'autres cultures, d'accéder à de nouvelles informations (Di Castri, 2002), de prévoir les catastrophes naturelles et donc d'en diminuer le risque, de prendre de meilleures décisions, etc. (Rodhain et Fallery, 2009).

Si les TIC ont été perçues comme une source d'avantages sur le plan économique et social, ce n'est que récemment que leur impact sur l'environnement est devenu une réelle préoccupation. Cette préoccupation est justifiée par les conséquences négatives générées par la production et l'utilisation des TIC. En effet, les produits numériques contiennent en général (Drezet, 2006):

- des substances toxiques qui nuisent à l'environnement et à la santé de l'homme comme l'antimoine, l'oxyde de baryum, le béryllium, le cadmium, le chlore, le brome, le plomb, le lithium, le mercure, les phosphores, l'arsenic, les retardateurs de flammes bromés, etc.

- des matériaux rares, chers et non renouvelables comme le cuivre, l'or, l'argent...

Il est important de signaler, à titre d'exemple, qu'un ordinateur de bureau à écran plat consomme, durant son utilisation, $350 \mathrm{~kg}$ en équivalent carbone (ADEME, 2007). Le bilan carbone s'alourdit avec le nombre des ordinateurs dans le monde qui ne cesse d'augmenter. Flipo (2007) mentionne qu'entre 1993 et 2000, le nombre de PC par habitant a augmenté de 181\%. Selon Gartner le nombre d'ordinateurs dans le monde passera d'un milliard à 2 milliard d'ici 2014 (Gartner, 2008).

Laquestion de la diffusion et de l'utilisation intensives des TIC etleurs conséquences négatives sur l'environnement a intéressé de nombreux chercheurs en écologie industrielle, en philosophie et en informatique. Cependant, nous remarquons que la littérature qui traite cette problématique en système d'information est relativement faible (Rodhain, 2005; Pensel, 2008; Rodhain et Fallery, 2009).

C'est pourquoi nous souhaitons, à travers cette recherche exploratoire, connaître les pratiques managériales en termes de gestion et d'utilisation responsable des TIC.

Ce papier de recherche présente dans une première partie une revue de la littérature sur l'impact écologique des TIC. Nous commençons par présenter un bilan écologique des TIC. Ensuite, nous détaillons ce qu'est une TIC écologique (Éco-TIC). Dans une deuxième section, nous présentons notre étude empirique qui se compose de deux études exploratoires. La première étude nous aide à connaitre comment se traduisent les éco-TIC dans les entreprises. Cette étude nous a menés sur une piste de recherche, selon nous, pertinente : celle de la gestion des déchets électroniques (e-déchets) dans les entreprises. Cette question a fait l'objet d'une deuxième étude et ce pour mieux comprendre la façon dont les entreprises gèrent ces e-déchets. A la fin de ce papier nous élaborons une conclusion de cette recherche. 


\section{Ecologie \& Technologies de l'Information et de la Communication (TIC) : une étude exploratoire sur les éco-TIC}

\section{Le recours aux Éco-TIC : Une manière pour réduire l'impact écologique des TIC?}

Selon Flipo (2007), les TIC véhiculent une image de légèreté et d'absence de friction. Elles ont été considérées pendant longtemps comme des solutions qui permettent de numériser et ainsi de dématérialiser les flux physiques. Leur impact sur l'environnement est peu abordé (Flipo, 2007) et se laisse cacher derrière des slogans tel que les produits immatériels, l'industrie du silicium, le zéro papier... (Fuchs, 2008; Rodhain et Fallery, 2009).

Nous commençons cette étude par dresser un bilan écologique des TIC. Le rareté de travaux en SI sur les TIC et écologie (Rodhain, 2005) nous pousse à creuser dans les revues informatiques et écologiques.

\subsection{Impact des TIC sur l'environnement : Un bilan écologique des TIC}

Dans le secteur informatique, le coût environnemental de la production, de l'utilisation et du recyclage est très important par rapport à d'autres secteurs (Flipo et Gossart, 2008). Christensen et al. (2007) notent qu'un grand nombre de problèmes environnementaux est lié à l'utilisation des TIC. Ces problèmes concernent la consommation énergétique en phases de production et d'utilisation mais aussi les effets de l'extraction de matières premières (cuivre, or, mercure) nécessaires à la production des TIC sur la santé des individus.

Nous procédons à la présentation de l'impact écologique des TIC sur les trois phases du cycle de vie de produit.

\subsection{1. ... en phase de production}

La production réclame une quantité de ressources considérable. A titre d'exemple, la fabrication d'un ordinateur de bureau avec son écran nécessite au moins 18 tonnes de matériaux divers, dont 240 kilos d'énergie fossile, 22 kilos de produits chimiques et 1500 litres d'eau (Drezet, 2006).

Kuehr et Williams (2003) ont publié le détail de la consommation énergétique fossile nécessaire à la production de différents sous composants d'un ordinateur personnel. Quelques exemples sont cités dans les deux tableaux suivants : 


\begin{tabular}{|c|c|}
\hline Electricité $(\mathrm{kWh})$ & 87 \\
\hline Combustibles fossiles (consommation directe) $(\mathrm{Kg})$ & 198 \\
\hline Combustibles fossiles (consommation globale) $(\mathrm{Kg})$ & 226 \\
\hline
\end{tabular}

Tableau 1 : Consommation énergétique pour la fabrication d'un écran plat (Kuehr et Williams, 2003)

\begin{tabular}{|l|c|}
\hline \multicolumn{1}{|c|}{ Fabrication de matériaux... } & Combustibles fossiles nécessaire par ordinateur (Kg) \\
\hline pour le boîtier de l'unité centrale & 21 \\
\hline pour le boîtier de l'écran & 22 \\
\hline pour les galettes de silicium & 17 \\
\hline de base (acier, plastique, verre) & 64 \\
\hline
\end{tabular}

Tableau 2 : Combustibles fossiles nécessaire pour la fabrication d'un ordinateur (Kuehr et Williams, 2003)

Le bilan n'est pas seulement dû à la production mais également à l'utilisation des TIC.

\subsection{2. ... en phase d'utilisation}

Berthoud et al. (2007) mentionnent que les TIC produisent autant de $\mathrm{CO}^{2}$ que les transports aériens. Econologie (Econologie, 2006) publie des statistiques montrant que la consommation énergétique mondiale en 2004 était d'environ 10000 millions de tonnes équivalent pétrole, « lissé sur un an et tout énergies confondues, cette consommation représente une puissance instantanée de 13,34 Milliards de $k W$, ramenée à la population terrestre " riche », cette consommation représente : $13,34 \mathrm{~kW}$ » soit 6 fois la consommation de 5/6 de la population terrestre. Cette énergie consommée se transforme en chaleur rejetée dans l'atmosphère.

L'infrastructure numérique demande par ailleurs à être entretenue et alimentée en énergie. J.A. Leitner ${ }^{101}$, de l'Environmental Protection Agency (EPA, Etats-Unis) estime que la consommation des TIC aux Etats-Unis en 2003 s'élevait à 3\% de la consommation totale d'électricité, tandis que Cremer $^{102}$ évalue la consommation allemande à 7\%. Des ordres de grandeur équivalents sont obtenus pour la Suisse avec un poids électrique des TIC de $10 \%$ sur la consommation totale (Flipo et Gossart, 2008).

Si quelques produits électriques ne consomment de l'énergie (électricité) que pendant leur effective utilisation, certaines TIC, comme les serveurs (web, de messagerie, de données...), en consomment sans arrêt. Principalement parce que ces serveurs doivent assurer une disponibilité et une accessibilité à tout moment. La consommation électrique de ces serveurs comprend aussi l'énergie

101. Cité par Flipo, F. et C. Gossart (2008). Infrastructure numérique et environnement : L'impossible domestication de l'effet rebond. Colloque international «Services, innovation et développement durable». 26-28 mars - Poitiers (France).

102. Cité par lbid. 


\section{Ecologie \& Technologies de l'Information et de la Communication (TIC) : une étude exploratoire sur les éco-TIC}

nécessaire au refroidissement des salles où ils sont installés. La consommation ne cesse d'augmenter d'autant que la nouvelle génération des TIC consomme beaucoup plus que la précédente. Ceci est dû à l'augmentation de la puissance et de la miniaturisation. Selon Flipo et Gossart (2008), cela pose des problèmes d'évacuation de chaleur et rend nécessaire d'énormes efforts sur la consommation. Berthoud et al. (2007) rajoutent que le coût de l'alimentation électrique dans les entreprises pourrait atteindre $30 \%$ du budget informatique dans les années à venir.

Ce pourcentage devrait d'ailleurs augmenter, car, comme l'explique Drezet (2006), la consommation augmente parallèlement avec la fréquence. L'auteur cite l'exemple des deux processeurs Intel et AMD qui sont passés respectivement de 18 W en 1996 à 130 W en 2006 et de 19 W en 1996 à 110 W en 2006. Selon le même auteur « l'augmentation de la consommation a engendré une augmentation de la dissipation calorifique (climatisation), des puissances des onduleurs et des nuisances sonores (à cause de la ventilation) » (P 3).

L'impact écologique des TIC ne s'arrête pas à la fin de leur utilisation, comme nous l'abordons dans le point suivant.

\subsection{3. ... en fin de cycle de vie}

Les produits TIC finissent tôt ou tard en déchets. Les déchets informatiques, appelés encore e-déchets, rentrent dans la catégorie 3 des DEEE (Déchets d'Equipements Electriques et Electroniques) (Directive_DEEE, 2003). L'élimination de ces déchets reste une menace, voire une catastrophe tant que les produits ne sont pas réintroduits dans les cycles naturels (Flipo et Gossart, 2008).

Le Programme des Nations Unies pour l'Environnement (PNUE) montre dans son bulletin d'alerte que ces déchets sont soit stockés chez les utilisateurs en tant que produits obsolètes, soit envoyés aux pays pauvres (parfois sous forme de don), soit recyclés. Flipo et Gossart (2008) notent que $50 \%$ à $80 \%$ des déchets des Etats-Unis sont exportés vers des destinations telles que la Chine et l'Inde. Selon DEWA/GRID-Europe ${ }^{103}, 70 \%$ des DEEE mis en décharge à New Delhi (Inde) proviennent des pays industrialisés (PNUE, 2005). Un documentaire ${ }^{104}$ réalisé par Silicon Valley Toxic Coalition montre l'état misérable de démantèlement et de recyclage des produits TIC. Exportés d'une manière illégale, ces e-déchets sont démontés avec des méthodes rudimentaires : à main nue en utilisant des gaz, des solutions acides, des fumées toxiques, etc. Les ouvriers sont des hommes, des femmes de tout âge mais aussi des enfants mineurs, souvent en bas âge. Ces activités ont des retombées sur la santé humaine et l'environnement. Ce bilan, exposé d'une manière brève, montre que les TIC représentent un secteur 
polluant. Néanmoins, depuis quelques années, les grandes marques informatiques et télécoms tentent de réduire l'impact écologique des produits. Les producteurs mettent de plus en plus à la disposition des professionnels et particuliers des produits dits écologiques. Cette vague de produits a été connue en premier sous le nom de « Green IT », ensuite sous le nom « éco-TIC». Que sont ces éco-TIC? Sont-ils vraiment efficaces du point de vue bilan écologique?

\subsection{Les éco-TIC, vers une informatique écologique?}

Dans cette partie, nous nous intéressons dans un premier temps à définir l'origine et la signification du terme éco-TIC. Ensuite, nous procédons à un essaie de classification que nous avons établi sur la base des différentes théories en rapport avec les éco-TIC. Nous terminons cette partie en présentant quelques effets de rebond qui interfèrent dans l'utilisation des éco-TIC.

\subsubsection{Qu'est ce qu'une éco-TIC ?}

Le terme éco-TIC a été créé en France le 12 juillet 2009 par la Commission Générale de Terminologie et de Néologie de l'informatique et des composants électroniques ${ }^{105}$. Cette commission a publié dans le Journal Officiel le terme « écotechnologie de l'information et de la communication » ou éco-TIC comme équivalent au Green IT (JORF, 2009). Selon la même source, les éco-TIC désignent les «techniques de l'information et de la communication dont la conception ou l'emploi permettent de réduire les effets négatifs des activités humaines sur l'environnement».

Traiter des questions en rapport avec les éco-TIC renvoie à étudier ces technologies aussi bien dans la phase de production (conception) que dans la phase d'utilisation (emploi).

Christensen et al. (2007) signalent que pendant la production des TIC, les questions en rapport avec la consommation d'énergie et de matière première nécessaires à la fabrication doivent être posées, sachant que certaines matières sont considérées comme rares, chères et non renouvelables (le cuivre, l'or, le nickel...).

Selon Pensel (2008), l'utilisation des TIC s'inscrit dans une perspective temporelle. La réduction de leur impact écologique pourrait se réaliser sur tout le cycle de vie. Pensel définit trois phases : avant (1), pendant (2) et après (3) l'utilisation.

(1) Avant l'utilisation, c'est-à-dire au moment de l'acquisition des produits, un management responsable des SI doit engager des choix d'achat et de renouvellement des outils matériels, ainsi que la maitrise de la facture énergétique (Christensen et al., 2007; Anderson et al., 2008; Pensel, 2008).

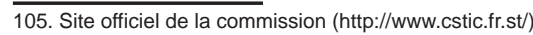




\section{Ecologie \& Technologies de l'Information et de la Communication (TIC) : une étude exploratoire sur les éco-TIC}

(2) Pendant l'utilisation de ces équipements, les entreprises et les individus doivent adopter un comportement responsable, notamment en ce qui concerne la consommation énergétique (Drezet, 2006; Berthoud et al., 2007; Christensen et al., 2007; Flipo et Gossart, 2008; Pensel, 2008).

(3) Enfin, les questions relatives aux TIC à la fin de vie doivent être soulevées. Ceci englobe les problèmes liés à la gestion des e-déchets (Berthoud et al., 2007; Christensen et al., 2007; Flipo et Gossart, 2008; Pensel, 2008; Flipo et al., 2009).

Les éco-TIC concernent donc plusieurs aspects aussi bien techniques que managériaux. "Faire de l'éco-TIC » peut se manifester sous plusieurs formes. Ceci nous a poussés à élaborer une classification des éco-TIC.

\subsubsection{Essai de classification des éco-TIC}

La classification des éco-TIC peut se faire de différentes manières. Nous pouvons, par exemple, considérer deux grandes catégories d'éco-TIC. (1) L'utilisation des TIC pour réduire l'impact écologique de l'activité humaine. (2) La conception et la mise en place des TIC de façon à ce qu'elles réduisent leur propre impact sur l'environnement.

Bordage (2009) propose une autre classification des éco-TIC qui se résume en trois catégories.

(1) Green IT 2.0 : L'utilisation des TIC pour réorganiser / optimiser des processus métiers en fonction de leur empreinte écologique.

(2) Green IT 1.5 : La réduction de l'empreinte écologique des infrastructures de communication et l'utilisation de ces outils pour réduire l'empreinte écologique de l'entreprise, notamment en réduisant les déplacements.

(3) Green IT 1.0 : La réduction de l'impact de l'informatique sur l'environnement. Nous pensons que cette classification est complexe et assez difficile à assimiler. Par exemple, il est difficile de savoir à travers cette classification si la réduction de l'empreinte écologique d'une activité fait partie du Green IT 2.0 ou 1.5.

Pour cette raison, nous avons tenté d'élaborer une classification plus simple, en se basant sur les travaux de Berthoud et al. (2007), Garretson (2007), Anderson et al. (2008), Pensel (2008), Bordage (2009) et Swanborg (2009). Nous pouvons catégoriser les éco-TIC en trois catégories, comme suit :

(1) L'ensemble des méthodes, logiciels et services qui réduisent l'impact du métier des entreprises sur l'environnement. Citons par exemple le e-commerce, le télétravail, la vidéoconférence et le e-Learning... Ces pratiques ont pour principal objectif d'éviter les déplacements et les transports physiques. 
(2) L'ensemble des méthodes et des logiciels qui ont pour objectif de réduire la consommation énergétique de l'infrastructure informatique. Dans cette catégorie on cite les « Green Data Center », l'informatique de nuage, la virtualisation des serveurs...

(3) L'ensemble des technologies de l'information et de la communication éco-conçues ou encore appelé les «TIC propres ». L'éco-conception est une manière préventive qui consiste à internaliser les problématiques écologiques dans le processus de production (Patingre et Vigneron, 2001), ainsi l'impact environnemental devrait être mesurable tout le long du cycle de vie du produit (fabrication, utilisation et gestion des déchets à la fin de vie).

La question que nous nous sommes posé au cours de ce travail de recherche est de savoir si le recours aux éco-TIC suffisait pour respecter l'environnement ? La section suivante éclaire certaines faces cachées des éco-TIC.

\subsubsection{L'effet de rebond des TIC}

Les TIC font l'objet d'une controverse : Selon Flipo (2007), les discours politiques montrent, par exemple, l'apport des TIC à « substituer les bits aux atomes » notamment pour la réduction de l'émission du $\mathrm{CO}^{2}$ lors des transports (à travers l'usage de la visioconférence, du commerce électronique, etc.). D'autres chercheurs montrent qu'un grand nombre de problèmes environnementaux est lié à l'usage des TIC.

Ainsi, selon Fuchs (2008), certains scientifiques soutiennent que le passage vers une " société de l'information » signifie que l'économie devient de moins en moins consommatrice en ressources, ce que l'auteur, à l'instar de Rodhain (2005), Flipo et Gossart (2008), Pensel (2008), Flipo et al. (2009) réfute, en livrant les exemples suivants :

- La dématérialisation de quelques services tels que la télé-facture, la télédéclaration fiscale ou l'e-payement, réduit l'utilisation du papier, or ceci ne peut être vrai que si l'utilisateur n'imprime pas ces documents électroniques.

- Le e-commerce, le télétravail, les vidéoconférences, le e-Learning et d'autres formes d'activités à distance évitent le déplacement des individus et donc réduisent leur empreinte écologique. Cependant, l'utilisation d'Internet crée de nouvelles relations sociales au niveau mondial et ainsi le besoin de se déplacer sur de longues distances se manifeste.

- Les produits électroniques ou téléchargeables (livre, journaux, logiciel, musique, vidéo...) remplacent les CD et les supports papiers. Néanmoins, la possibilité d'imprimer ou de graver ces données sur des supports physiques existe dans ce cas également.

Ainsi, la dématérialisation génère souvent un « effet rebond » (Flipo et al., 2009), c'est-à-dire qu'un progrès technologique n'est pas associé, comme attendu, 


\section{Ecologie \& Technologies de l'Information et de la Communication (TIC) : une étude exploratoire sur les éco-TIC}

à une diminution de la consommation, mais une augmentation, car le gain de productivité lié au progrès technologique conduit le consommateur à consommer plus et non pas moins (Schneider, 2003).

De cette première section, nous pouvons constater que les éco-TIC semblent couvrir plusieurs champs d'application. Afin de savoir comment les entreprises traduisent le concept d'éco-TIC, nous avons mené une étude empirique qui sera présentée dans la section suivante.

\section{Les éco-TIC dans les entreprises françaises}

Nous avons démarré ce travail de recherche avec l'objectif d'énumérer les pratiques éco-TIC dans les entreprises françaises. Et ensuite, nous avons fait émerger du terrain la ou les pratiques les plus problématiques. L'étude empirique s'est déroulée en deux étapes :

Une première étude exploratoire a consisté à connaître les pratiques en matière d'éco-TIC dans les entreprises françaises. Pour cela, nous avons mené des entretiens ouverts non directifs auprès de personnes impliquées dans des démarches éco-TIC.

Cette étude a mis en lumière une problématique intéressante celle de la gestion des déchets électroniques (e-déchets) dans les entreprises. Afin de mieux connaître les stratégies mises en place par les entreprises pour la gestion des e-déchets, nous avons mené une deuxième étude exploratoire sur cette problématique. La deuxième étude se base sur des entretiens directifs avec des questions directes auprès de responsables environnementaux.

\subsection{Les pratiques éco-TIC}

L'objectif de cette première étude est de connaître les différentes pratiques en matière des éco-TIC dans les entreprises françaises. Nous commençons par présenter les modalités de cette recherche (échantillon, méthode de collecte et d'analyse des données). Ensuite, nous présentons les principaux résultats. Enfin, nous terminons cette partie avec une conclusion.

\subsubsection{Modalités de la première étude exploratoire}

Pour répondre à notre premier objectif, nous avons mené une étude préexploratoire sur un échantillon de convenance de sept personnes impliquées dans des démarches d'éco-TIC.

\subsubsection{Echantillon}

Nous avons établis un premier contact avec deux interviewés lors d'une journée professionnelle sur les " éco-TIC ». Les autres contacts ont été repérés à travers le réseau professionnel Viadeo. Nous les avons choisit en fonction de 
leur participation aux hubs « Green IT ». Nous avons envoyé 50 messages sur Viadeo. 5 seulement ont répondu favorablement.

Les entreprises de notre échantillon se répartissent comme suit : trois SS2I, une grande chaîne de distribution, deux organisations de service publiques et une société qui opère dans les services bancaires. Nous avons sollicité des DSI, des informaticiens, un responsable d'affaire et un consultant « Green IT » (en free lance).

\subsubsection{Méthode de collecte de données}

Nous avons mené, dans cette première étude, des entretiens non directifs. Car, comme le souligne Igalens et Roussel (1998), les entretiens conviennent particulièrement à des sujets mal connus.

Nous avons établi un guide d'entretien avec des questions ouvertes, laissant aux interviewés le soin de raconter leur pratiques et stratégies éco-TIC. Les entretiens se sont déroulés en juin 2009. Leur durée varie entre 45 et 10min (avec une moyenne de $27 \mathrm{~min}$ ).

\subsubsection{Méthode d'analyse de données}

Nous avons retranscrit ces entretiens en intégralité avant d'entamer le processus d'analyse de données. Savoie-Zajc (2000), en se basant sur le travail de Tesch ${ }^{106}$, définit l'analyse de données comme un processus qui requiert un effort explicite d'identification de thèmes, de construction des hypothèses.

Pour identifier les thèmes qui ressortent de ces entrevues, nous avons effectué une première lecture de l'ensemble des données. Ensuite, lors de la deuxième, lecture nous avons procédé à un travail de sur-lignage et de remarques sur les marges. Ceci a permis de dégager les thèmes transversaux suivant :

1. La motivation pour l'adoption des éco-TIC,

2. Les stratégies mises en place,

3. Les technologies mises en place.

Nous avons codé ces données sous Microsoft Excel 2007 selon la méthode de regroupement conceptuel (Miles et Huberman, 1994) . La partie suivante énumère les principaux résultats de cette étude exploratoire.

\subsubsection{Résultats}

Comme nous avons mentionné ci-dessus, nous avons pu connaître les différentes motivations quant à l'adoption des éco-TIC, nous avons aussi pu définir les différentes stratégies et technologies employées dans le cadre de la mise en place des éco-TIC. Nous présentons des verbatims illustrant les thématiques présentées :

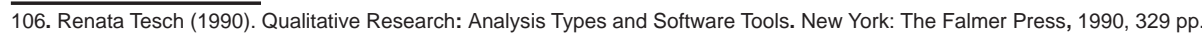




\section{Ecologie \& Technologies de l'Information et de la Communication (TIC) : une étude exploratoire sur les éco-TIC}

(1) La motivation pour l'adoption des éco-TIC

Six interviewés signalent que l'adoption des éco-TIC est surtout stimulée par des raisons économiques (économies d'énergie). Une personne pense qu'inscrire l'entreprise dans une démarche verte permet « de fédérer des collaborateurs autours de [notre entreprise], convaincre les nouveaux consultants par rapport à notre problème (...) puis justement de montrer à notre échelle qui est une structure à mode de fonctionnement durable que ça peut fonctionnens.

(2) Les stratégies mises en place

Nous avons mis en évidence trois stratégies en rapport avec les éco-TIC.

La première concerne l'achat des produits numériques. Les entreprises adoptent une démarche d'achat responsable sur la base des certifications internationales ou des écolabels «Les entreprises commencent à le faire grâce notamment à un certain nombre d'écolabel ».

« Je fais une sélection de matériel le plus vert possible, avec beaucoup de test en amont et de comparaison pour trouver le matériel le plus performant énergétiquement»

«On a introduit une charte d'achat responsable entre le groupe et les prestataires avec lesquels on contractualise ». Pour acheter d'une manière responsable quelques entreprises se fondent aussi sur des informations du net « Mais aussi en passant par des sites internet ou des spécialistes qui offrent régulièrement des tests ou des comparatifs sur les produits écologiques».

La deuxième concerne la gestion du papier et des impressions. Certaines entreprises mettent en place des projets de sensibilisation auprès des collaborateurs pour une meilleure gestion du papier et de l'encre. « II y a une politique de papier responsable (...) de recourt au papier recyclé ou issu de forêts gérées durablement ». Dans d'autres cas, les collaborateurs prennent l'initiative de gérer leurs propres impression " Par rapport la réduction de l'utilisation du papier, c'est surtout des initiatives personnelles».

La troisième concerne la gestion des déchets électroniques. Trois entreprises de l'échantillon ont évoqué la question des e-déchets. «On a commencé à travailler sur les déchets, c'est-à-dire le recours à des filières de déchets électroniques». « La gestion des déchets est un chantier d'avenir ». " Je sais qu'on a fait appel à un Broker, les Brokers sont des sociétés qui reprennent le matériel, je pense qu'on a une obligation, tout ce qui est matériel informatique devrait être recyclé ».

(3) Les technologies mises en place

A travers les entretiens, nous avons identifié trois techniques en relation avec les éco-TIC qui sont la virtualisation, le Green DataCenter et l'utilisation des applications en réseaux. 
- La virtualisation est une technique qui consiste à regrouper dans un serveur physique plusieurs serveurs logiques sous forme d'applications logicielles. Il s'agit de « diminuer le nombre de serveurs et de faire en sorte que ce que l'on a soit plus efficacement utilisé ». Selon un responsable DSI, un projet de virtualisation est « un projet lié au développement durable ou à la préservation des ressources! C'est plus une question d'économie par rapport aux serveurs que nous avons à gérer ».

- Le Green Data Center est un entrepôt de stockage, de gestion et de diffusion de données où l'éclairage, l'électricité et les systèmes informatiques sont conçus pour une efficacité énergétique maximale et un impact environnemental minimal. La construction d'un centre de données vert comprend des technologies de pointe et des stratégies comme : minimiser les empreintes des bâtiments, minimiser l'énergie dépensée pour le refroidissement de l'infrastructure, l'utilisation des technologies d'énergie alternative comme l'énergie photovoltaïque, pompes à chaleur, et le refroidissement par évaporation...

- Le recours à des applications en réseaux, pour réduire l'impact de la logistique et réduire les transports. Par exemple « la mise en œuvre d'un portail collaboratif et d'outil de Web conférence (...) pourraient permettre de réduire le déplacement des collaborateurs ».

Notons que nous nous sommes arrêtés au 7ème entretien, car nous avons remarqué qu'au bout du 4ème entretien nous avons atteint la saturation. C'est-àdire que les pratiques éco-TIC se répètent. En effet, lors du deuxième entretien, nous avons interrogé un consultant Green IT. Ce dernier a travaillé pendant deux ans dans ce domaine. Durant son interview, il a cité la majorité des pratiques éco-TIC.

\subsubsection{Conclusion}

D'après cette première étude, il semble qu'il existe plusieurs manières de mettre en place des éco-TIC. L'étude montre que chaque entreprise de notre échantillon les choisi en fonction de ses besoins, de ses moyens et aussi de sa motivation. Nous avons également montré que la problématique des e-déchets pourrait être une question de recherche pertinente pour 2 raisons :

(1) Les recherches en informatique et en écologie industrielle montrent qu'on ne peut pas arrêter le progrès électroniques, la production informatique ne fait qu'augmenter, ainsi que la consommation. Nous ne pouvons pas arrêter ce progrès. S'il des solutions peuvent exister pour diminuer l'impact des TIC sur l'environnement ca sera des solutions en gestion des déchets. Pour passer d'une économie linéaire à une économie cyclique, il serait nécessaire d'injecter dans les processus de production ce qu'on appelle des matières « premièressecondaires ». II s'agit de matière issue des produits recyclés. 


\section{Ecologie \& Technologies de l'Information et de la Communication (TIC) : une étude exploratoire sur les éco-TIC}

(2) Les interviewés soulignent l'importance de la gestion des e-déchets: "Ces dernières années on a une croissance d'à peu près 15\% par an de la puissance électrique consommée dans les data center, au niveau du matériel informatique. C'est dû à une croissance de besoin, c'est dû à une extension de périmètre de ce que l'on gère mais ça peut continuer. Enfin il $n$ y a pas, pour l'instant on ne voit pas de raison même on utilisant derrière du matériel moins consommateur, plus efficace. On voit pas de raison que cette croissance ne s'arrête pas ».

" On va parler de prolonger la durée de vie du matériel, durée d'utilisation du matériel, ensuite on va parler de matériels d'occasion reconditionnées, donner une seconde vie à du matériels d'occasion donc prolonger d'une autre façon sa durée de vie. Un grand problème, un chantier d'avenir ».

«Tout ce qui est matériel informatique devrait être recyclé ».

Trois interviewés signalent l'importance des deux dernières catégories, c'est-àdire de réaliser des économies d'énergies sur la phase d'utilisation mais aussi d'utiliser des TIC propres. II s'agit en fait, d'une part, de prolonger la durée de vie du matériel et sa durée d'utilisation et d'autre part de reconditionner du matériel d'occasion, c'est-à-dire de donner une seconde vie au matériel d'occasion. Cependant, ce dernier point n'est pas abordé par les autres personnes interrogées.

L'intérêt suscité dans la littérature aux questions des e-déchets (PNUE, 2005; Drezet, 2006; Flipo, 2006; Berthoud et al., 2007; Flipo, 2007; Flipo et Gossart, 2008) ne semble pas secouer les managers des entreprises. Nous jugeons pertinent de nous diriger dans cette voie afin de connaître la manière dont les entreprises gèrent leurs e-déchets. C'est pour cela que nous avons mené une deuxième étude dont l'objectif est de connaître les différentes stratégies de gestion des e-déchets dans les entreprises. La section suivante détaille les modalités et les résultats de cette étude.

\subsection{La gestion des e-déchets}

Lors de nos recherches sur les e-déchets, nous avons choisi de nous orienter sur l'efficacité des filières de traitement de ces déchets en France. Lorsque nous avons compris qu'il existait un décalage dans la mise en place des filières de traitement des déchets d'équipements électriques et électroniques (DEEE) ménagers et professionnels, nous avons décidé de contacter les entités qui sont à la source de la filière, c'est-à-dire les entreprises françaises.

L'objectif principal de notre travail est de faire un « état des lieux » de leur connaissance de ce problème, de leur démarche et de leurs actions liées à cet enjeu pour ensuite faire des propositions d'améliorations niveau organisationnel. 


\subsubsection{Modalités de la seconde étude exploratoire}

Nous sommes partis d'un constat et d'une réflexion initiale pour aller jusqu'au déroulement des entretiens en passant par le choix de l'échantillon et l'élaboration du guide d'entretien. Les réponses des 20 entreprises consultées ont été exploitées et analysées afin de pouvoir en tirer des conclusions intéressantes et ainsi chercher des pistes d'amélioration dans le traitement des DEEE des entreprises françaises.

\subsubsection{Echantillon}

Pour la consultation des entreprises, étant limités par le temps, il nous a fallu définir des critères judicieux pour obtenir un échantillon de résultats exploitables. C'est pourquoi, nous avons décidé de restreindre géographiquement notre enquête à l'échelle régionale et plus précisément au Languedoc-Roussillon, ceci pour un aspect pratique en cas de nécessité de déplacement et de rencontre. Dans un second temps, nous avons réfléchi aux types d'entreprises vers lesquels nous pouvions nous orienter. Se focaliser sur un ou plusieurs secteurs d'activité définis tels que l'industrie informatique nous a semblé trop restrictif et non représentatif des entreprises françaises dans leur ensemble. Par contre, les entreprises certifiées ISO $14001^{107}$ représentent un panel intéressant d'entreprises pour notre enquête puisque tous les secteurs d'activité peuvent être concernés et que ce sont des sociétés qui ont déjà choisi d'adopter une démarche environnementale.

Etant donné qu'il n'existe pas de liste exhaustive des entreprises certifiées ISO 14001 en Languedoc-Roussillon, nous avons obtenu les informations à l'aide d'internet et du Qualipôle de Montpellier. Parmi les 60 sociétés répertoriées, nous avons choisi un échantillon de 20 entreprises à consulter.

\subsubsection{Méthode de collecte des données}

Pour mener à bien la consultation des entreprises, nous avons interviewé les personnes responsables du suivi de la norme dans chaque établissement en suivant un guide d'entretien préétabli. Ce guide d'entretien s'articulait autour de 4 thèmes relatifs à la norme ISO 14001, aux problèmes des DEEE et de la législation, ainsi qu'au thème des DEEE dans la norme ISO 14001 qui abordait plus spécifiquement la démarche adoptée par l'entreprise concernant la gestion des DEEE et plus particulièrement des é-déchets.

Suite à un premier échantillon de 6 entreprises et une rapide analyse des résultats obtenus, nous nous sommes concertés pour apporter de légères modifications sur le contenu et la forme du guide d'entretien.

107. Est une norme internationale reposant sur une démarche volontaire des entreprises qui souhaitent maîtriser et réduire les impacts sur l'environnement causés par leur activité. Cette norme permet de mieux connaître les impacts environnementaux générés par les activités de l'organisme, de garantir le respect de la réglementation et d'être à même d'anticiper les évolutions de cette dernière et d'adopter une logique d'amélioration continue de réduction des impacts environnementaux. 


\section{Ecologie \& Technologies de l'Information et de la Communication (TIC) : une étude exploratoire sur les éco-TIC}

\subsubsection{Méthode d'analyse de données}

Les entretiens ont été enregistrés sur dictaphone. Chaque interview a été réécoutée, analysée et les éléments importants ont été retranscrits dans un tableau de résultats. Ceci a été effectué en suivant toujours les thématiques instaurées dans le guide d'entretien.

Lorsque l'objectif des 20 entreprises consultées a été atteint, nous avons étudié l'échantillon dans son ensemble. Pour chaque thématique, en fonction du pourcentage de réponse à chaque question, de l'hétérogénéité de celles-ci et de leur intérêt, nous avons déterminé si il était judicieux de l'exploiter et si oui, quels points il fallait développer en particulier.

C'est donc en suivant cette démarche que nous avons abouti aux résultats développés ci-dessous.

\subsubsection{Résultats de la deuxième étude}

Après une analyse détaillée de l'ensemble de l'échantillon, nous avons pu identifier, d'une part (1) pourquoi les entreprises gèrent ou non leurs e-déchets, et d'autre part, (2) les différentes stratégies de gestion des e-déchets.

(1) Nous avons constaté que 13 entreprises sur 20 gèrent leurs DEEE.

Parmi les 7 entreprises restantes, 4 n'ont aucune démarche concernant les DEEE. Une société a une certification sur ses missions et non sur ses bâtiments. C'est pourquoi la gestion des déchets ne rentre pas dans la norme appliquée. Les 3 autres entreprises, qui ne gèrent pas leurs DEEE, ont prétendu que leur quantité produite était trop faible.

Trois entreprises n'ont pas été comptabilisées car elles ne relèvent pas des situations précédentes. En effet, la première n'a pas souhaité répondre à notre enquête pour cause de confidentialité. Les DEEE de la seconde sont récupérés par le fournisseur directement (on considère donc qu'ils ne gèrent pas leur DEEE directement). Et enfin, la gestion du parc informatique de la troisième est entièrement sous-traitée de l'achat à la fin de vie.

Par conséquent, nous pouvons dire que la principale raison de la non-gestion des DEEE au sein des entreprises de cet échantillon est la trop faible quantité de déchets électroniques produits. Soit il n'est pas jugé nécessaire de mettre en place un cadre administratif pour une faible quantité de déchet mais cela n'empêche pas leur bonne gestion lorsqu'il y en a. Soit la faible quantité produite peut être à l'origine d'un manque de conscience de la toxicité de ces déchets et ainsi entraîner un comportement irresponsable comme les jeter avec les ordures ménagères. Ensuite, nous constatons que seulement 2 entreprises sur les 13 gérant leurs DEEE le faisaient avant d'être certifiées. En extrapolant de façon 
osée voire abusive nous pourrions penser que seulement 10\% (2 entreprises sur 20) des entreprises non certifiées ISO 14001 en France ont une démarche de gestion de leurs DEEE. Certes, l'échantillon est trop restreint pour tirer de telles conclusions, mais cela nous laisse présager quand même un faible taux de gestion de DEEE au sein des entreprises françaises. 7

(2) Lorsque les entreprises ont décidé de gérer leurs DEEE, elles adoptent toutes le même fonctionnement. Dans un premier temps, elles trient les déchets sur le site soit en comptant sur le comportement responsable de chaque employé, soit, comme c'était le cas pour une des sociétés interviewées, par l'intermédiaire d'un centre de tri présent sur le site.

Ensuite, les entreprises font appel à des prestataires agréés qui récupèrent les DEEE et les traitent (recyclage et/ou élimination). Suivant, la capacité de stockage et la quantité produite de chacune, l'enlèvement est plus ou moins fréquent. II est délicat d'annoncer une échelle de production annuelle puisque celle-ci varie fortement en fonction des activités et des changements de l'entreprise (ex : renouvellement du parc informatique), les chiffres annoncés par nos interlocuteurs s'étendaient de $40 \mathrm{~kg} / \mathrm{an}$ à 1t/an. Une personne a souligné le fait que les DEEE passaient d'abord dans les mains des employés de leur service informatique qui récupéraient les pièces encore utilisables avant de les jeter, nous pouvons qualifier cela d'une étape de réutilisation avant les étapes de recyclage et d'élimination.

Pour finir, parmi les entreprises qui font appel à des prestataires, 9 appliquent un suivi de l'activité de ces derniers et du devenir de leurs DEEE selon 3 niveaux:

- les prestataires envoient des documents d'information sur leur activité et émettent des BSD (Bordereaux de Suivi de Déchets) détaillés après chacune de leur intervention (6 entreprises sur 9),

- l'entreprise recevant le service visite une fois ou régulièrement le site du prestataire (2 sur 9),

- l'entreprise recevant le service audite le prestataire (1 sur 9).

Nous résumons ces principaux résultats dans le tableau suivant :

\begin{tabular}{|l|c|}
\hline Stratégie adoptée & N entreprises (/20) \\
\hline $\begin{array}{l}\text { Gestion des e-déchets } \\
\text { depuis la certification }\end{array}$ & 13 \\
\hline $\begin{array}{l}\text { Gestion des e-déchets avant } \\
\text { la certification }\end{array}$ & 2 \\
\hline Recours à des prestataires & 13 \\
\hline Suivi des e-déchets & 9 \\
\hline
\end{tabular}

Tableau 4 : Stratégies éco-TIC adoptées 


\section{Ecologie \& Technologies de l'Information et de la Communication (TIC) : une étude exploratoire sur les éco-TIC}

\subsubsection{Conclusion}

De cette étude nous remarquons qu'il existe différents niveaux de gestion des e-déchets dans les entreprises:

Niveau « passif » : L'entreprise ne s'occupe pas de la gestion des e-déchets.

Niveau « moyen » : L'entreprise délègue la gestion e-des déchets à un prestataire et visite son site pour le suivi de la prestation.

Niveau « actif » : L'entreprise délègue la gestion des e-déchets à un prestataire et audite son site pour le suivi de la prestation.

\section{Conclusion, limites et perspectives de la recherche}

A travers ce travail nous avons tenté de faire un état des lieux des liens pouvant exister entre TIC et écologie. Nous avons mené une réflexion théorique sur l'impact écologique des TIC. La rareté des travaux en systèmes d'information nous a obligé à explorer d'autres revues scientifiques en informatique et écologique industrielle notamment, ainsi que des rapports des grandes organisations nationales et internationales, notamment l'ADEME.

Nous constatons que l'industrie numérique requiert des matières premières chères, précieuses et généralement non renouvelables. Avec le rythme de développement et de renouvèlement des produits TIC, les ressources naturelles s'épuisent. Le marché informatique et télécom se développe très rapidement. La taille des composantes devient de plus en plus petite, leur puissance augmente, et leur durée de vie se réduit. La consommation énergétique de ces produits ne peut être ignorée, et la question de leur devenir doit être traitée avec plus de sérieux.

L'étude exploratoire, que nous avons mené lors de cette recherche, nous a conduit, dans un premier temps, à mieux connaître comment se traduisent les éco-TIC dans les entreprises. Ensuite, à cadrer une question de recherche que nous jugeons pertinente. II s'agit de la gestion des infrastructures numériques en fin de vie.

Atravers la deuxième étude nous avons pu identifier les motivations et les différentes stratégies de gestion des e-déchets. Les résultats de cette enquête font nous poser la question de l'existence d'une réelle conscience environnementale dans les entreprises. En effet, premièrement, nous avons compris que la certification était surtout un moyen de soigner la bonne image de l'entreprise tout en réduisant son impact sur l'environnement. Aussi, la faible proportion d'entreprise faisant un suivi actif de leurs déchets informatiques montre un manque d'intérêt vis-à-vis de cette problématique. 
Enfin, étant donné que la quasi-totalité des entreprises interrogées ne géraient pas leurs déchets informatiques avant d'être certifiées, on peut se demander ce qu'il en est réellement des entreprises françaises en générale. La taille de notre échantillon ne nous permet de répondre à cette question. C'est pourquoi, nous pensons élargir cette étude sur un échantillon plus grand en nous adressant aussi aux entreprises spécialisées dans la collecte des e-déchets.

\section{Références}

ADEME. (2007). «Bilan Carbone ${ }^{\circledR}$ Entreprises et Collectivités : Guide des facteurs d'émissions.» Retrieved Version 5.0, from http://www2.ademe.fr/servlet/getBin?name= CD6902D1AAFD8740470C44C136A32C451169215062423.pdf.

Anderson, G., et al. (2008). «Power Efficiency and Sustainable Information Technology.» Intel Technology Journal 12 (4): 303-311.

Berthoud, F., et al. (2007). Comment se diriger vers une informatique durable? La septième édition des JRES (Journées Réseaux). 20-23 novembre - Strasbourg.

Bordage, F. (2009). «Green IT : tentative de définition.» from http://www.greenit.fr/article/ acteurs/green-it-tentative-de-definition.

Breuil, H., et al. (2008). «TIC et Développement durable.» Conseil général de l'environnement et du développement durable $\left(\mathrm{N}^{\circ}\right.$ 005815-01) \& Conseil général des Technologies de l'Information ( ${ }^{\circ}$ IV-1.6-2008): $96 \mathrm{pp}$.

Christensen, T. H., et al. (2007). «Greening the Danes? Experience with consumption and environment policies.» Journal of Consum Policy 30(91-116).

Di Castri, F. (2002). Les conditions gagnantes du développement durable. Colloque International Francophonie et développement durable, 11, 12 et 13 mars - Dakar, Sénégal.

Directive_DEEE (2003). Directive 2002/96/CE du Parlement Européen et du Conseil du 27 janviēr 2003 relative aux déchets d'équipements électriques et électroniques (DEEE). Journal officiel de l'union européene. 13/02/2003.

Drezet, E. (2006). Les faces cachées de l'informatique : énergie et déchets. Admin06Resinfo 05/2006. A. CNRS-CRHEA.

Econologie (2006). «L'econologie en chiffres et en bref « Econologie: http://www. econologie.com/la-consommation-mondiale-d-energie-articles-3282.html.

Flipo, F. (2006). Projet E-dechets Ecologie des infrastructures numériques. Bretagne, INT - ENST.

Flipo, F. (2007). «L'infrastructure numérique en question.» Entropia 3: 67-85.

Flipo, F. et C. Gossart (2008). Infrastructure numérique et environnement : L'impossible domestication de l'effet rebond. Colloque international «Services, innovation et développement durable». 26-28 mars - Poitiers (France).

Flipo, F., et al. (2009). Technologies numériques et crise environnementale : peut-on croire aux TIC vertes ?, Université de Caen Basse-Normandie - Telecom et Management Sud Paris - Telecom Bretagne: 213.

Fuchs, C. (2008). «The implications of new information and communication technologies for sustainability.» Environ Dev Sustain 10: 291-309.

Garretson, C. (2007). "Inside a green data center." Network World 24(43): 49.

Gartner. (2008). "Gartner Says More than 1 Billion PCs In Use Worldwide and Headed to 2 Billion Units by 2014." Garthner Newsroom, from http://www.gartner.com/it/page. jsp?id=703807

Igalens, I. et P. Roussel (1998). Méthodes de recherche en gestion des ressources humaines. Paris, Economica. 207 pp.

JORF. (2009). «écotechniques de l'information et de la communication (Définition).» 


\section{Ecologie \& Technologies de l'Information et de la Communication (TIC) : une étude exploratoire sur les éco-TIC}

JORF (Journal Officiel de la République française), from http://www.legifrance.gouv.fr/ affichTexte.do?cidTexte $=$ JORFTEXT000020835844\&dateTexte $=$.

Kuehr, R. et E. Williams (2003). Computers and the environment: understanding and managing their impacts, Kluwer Academic. 300 pp.

Miles, M. B. et A. M. Huberman (1994). Qualitative data analysis, CA: Sage. 338 pp.

Patingre, J.-F. et J. Vigneron (2001). Eco-Conception - Concept, Méthodes, Outils, Guides Et Perspectives.

Pensel, J.-L. (2008). Quelles perspectives pour l'Informatique responsable ? AIM. 13-14 décembre - Paris-Dauphine.

PNUE. (2005). «Les Déchets Electroniques, la face cachée de l'ascension des technologies de l'information et des communications.» PNUE (Programme des Nations Unies pour l'Environnement), from http://www.grid.unep.ch/product/publication/download/ ew_ewaste.fr.pdf.

Rodhain, F. (2005). Appel à des recherches sur l'Ecologie et les TI. Xème Colloque de l'AIM. 22-23 septembre - Toulouse.

Rodhain, F. et B. Fallery (2009). ICT and Ecology: In favour of research based on the responsibility principle. 4th Mediterranean Conference on Information Systems. 25-27 Septembre - Athens, Greece.

Savoie-Zajc, L. (2000). "L'analyse de données qualitatives pratiques traditionnelle et assistée par le logiciel NUD.IST." Recherches qualitatives 21: 99-123.

Schneider, F. (2003). «L'effet rebond.» Ecologiste. Edition française de The Ecologist 4(3): 45.

Swanborg, R. (2009). “Where Green IT Is No Fad; Raytheon's Green IT initiative started with data center virtualization. But today's Green IT initiatives encompass system development and business efficiency." Framingham 22(18). 